\title{
The aims and scope of WikiJournal of Science
}

\author{
WikiJournal of Science editorial board ${ }^{1 *}$
}

\begin{abstract}
WikiJournal of Science is an open access, peer reviewed journal, free of publication charges for its authors. It has Wikipedia-integration as a key feature and aims to encourage and recognise contributions to Wikipedia by academics. It is a sister journal to the established WikiJournal of Medicine, and covers science, technology, engineering and mathematics. This editorial will discuss the current aims and future scope of the journal, as well as the WikiJournal format in general.
\end{abstract}

\section{The Wikipedia-academia divide}

Wikipedia plays an increasingly important role in scientific research and teaching, by providing a universally accessible compilation of well-known ideas and information. As a result, a typical Wikipedia article on a scientific subject has about as many viewers every day as a typical scientific article has readers over its lifetime. ${ }^{[1]}$ However, relatively few academics write in Wikipedia, and the coverage of many topics could greatly benefit from more attention by specialists. ${ }^{[2][3][4]}$ Indeed most articles are 1-2 paragraph stubs. ${ }^{[5]}$

Researchers currently have little incentive to contribute to Wikipedia, largely because such contributions do not count as academic publications, the main drivers of academics' careers. ${ }^{[6]}$ Further, Wikipedia is not an ideal source to cite in scholarly publications, since it is an evolving, tertiary source written by large numbers of mostly pseudonymous or anonymous authors. ${ }^{[7]} \mathrm{A}$ main aim of the WikiJournals is to address this issue, by allowing academics to write Wikipedia articles that also count as bona fide scholarly publications. This builds on experimentation in dual-publishing academic articles into Wikipedia by several journals, such as PLOS Computational biology, ${ }^{[8]}$ Gene, $^{[9]}$ RNA Biology, $^{[10]}$ and Open Medi-

\footnotetext{
${ }^{1}$ Board members at time of writing

*Author correspondence:

Thomas Shafee

T.Shafee@LaTrobe.edu.au

ORCID: 0000-0002-2298-7593

Licensed under: CC-BY

Published 01-06-2018
}

cine $^{[11]}$ Conversely, expert-written online encyclopedias such as Scholarpedia ${ }^{[12]}$ and Citizendium ${ }^{[13]}$ have remained entirely separate from Wikipedia, and this has severely limited their reach and scope.

\section{WikiJournals}

A primary feature of WikiJournals is their focus on dualpublication of review articles in the journal and in Wikipedia. In particular, WikiJournals publish a stable, citeable version of record as well as seeing a copy in Wikipedia that serves as a highly visible, living version (Figure 1). ${ }^{[1]}$ WikiJournals are formatted and published using the same MediaWiki software as Wikipedia. This enables suitable material to be integrated from WikiJournal articles into Wikipedia, including Lysine and ShK toxin. ${ }^{[14][15]}$ Similarly, Wikipedia articles can be submitted to WikiJournals for peer review, for example Radiocarbon dating and Spaces in mathematics. ${ }^{[16][17]}$ In

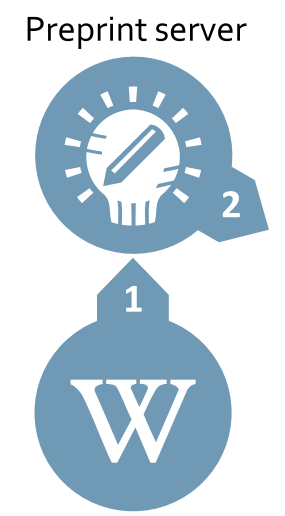

Wikipedia as preprint

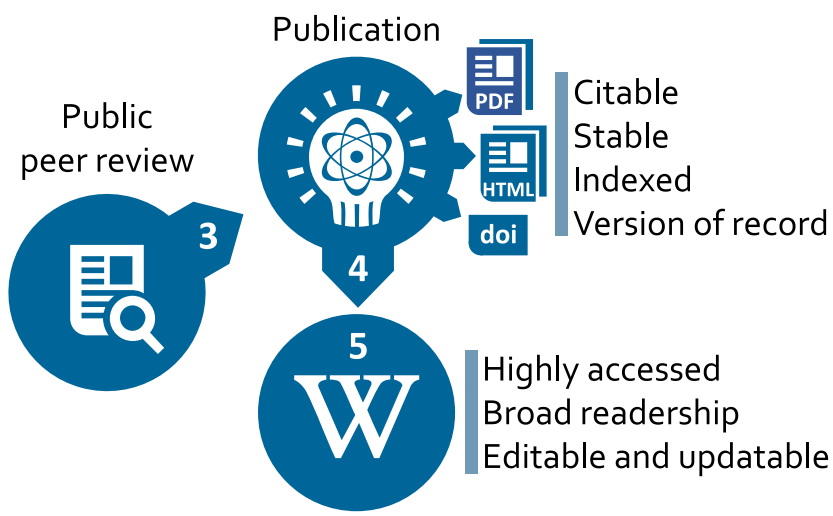

Wikipedia-integration
Figure 1 | Outline of publication process for Wiki.J.Sci. Articles may be written from scratch or adapted from existing Wikipedia material. Submission via the preprint server is recommended. External, academic peer review is organised by the journal editors. If published, suitable material is integrated back into Wikipedia to improve the encyclopedia. 
both cases, these broad, review-style articles need to conform to both scholarly best practices and Wikipedia's rules. WikiJournals can also publish original research articles that are not destined to be integrated into Wikipedia, such as A card game for Bell's theorem and its loopholes but can nevertheless benefit from the collaborative editing that MediaWiki enables. ${ }^{[18]}$

As academic journals, WikiJournals strive to follow recognised best practices in scholarly publishing, especially features advocated by the open access movement. ${ }^{[19][20][21]}$ In particular, we want to encourage open and collaborative reviews, ${ }^{[22]}$ the use of preprints, ${ }^{[23][24]}$ and reviews based on scientific merit, rather than perceived novelty or importance. ${ }^{[25]}$ In order to achieve this,

1. Articles are openly accessible and reusable, free of charge to authors and readers alike, under permissive Creative Commons licenses (libre open access).

2. Peer reviewers' comments are publicly accessible, and comments from the public are welcome.

3. The WikiJournals are independent, not-for-profit journals, supported by the Wikimedia Foundation and volunteer editorial teams.

The format was pioneered by WikiJournal of Medicine, ${ }^{[26]}$ which itself took inspiration from earlier experiences in Wikipedia-integrated publishing on medical and biomedicine topics. ${ }^{[1][11]}$ The model is now being extended by WikiJournal of Science to cover STEM topics more broadly.

\section{WikiJournal of Science: a broad scope Wikipedia-integrated journal}

Wiki.J.Sci. aims to achieve a broad scope across the STEM fields, and to become the main journal of its kind in these fields, including biology, physics, chemistry, technology, engineering, and mathematics. In addition to publishing on topics that were previously absent or underdeveloped in Wikipedia, it also allows the less common format of Wikipedia-first publication, treating Wikipedia as a preprint server.

In addition to review articles, Wiki.J.Sci. also publishes other formats that are not able to be integrated directly into Wikipedia, but still benefit from the libre open access model. Running the journal on Wikimedia software is well suited to collaborative, transparent, versioned, open writing. It also enables the journal to run on a very low budget, allowing articles to be processed without asking authors to pay fees. This contrasts with the "predatory journal" model that has proliferated to take advantage of the open-access movement. ${ }^{[27]}$

\section{Development and future of the format}

The unique features of the WikiJournal format have prompted us to codify them in a statement of publication ethics. ${ }^{[28]}$ This statement lays out principles for handling Wikipedia-integrated content, in addition to standard publication ethics. The editorial board encompasses a combination of academic research and publishing experience, as well as knowledge of Wikipedia's workings. We are therefore well-positioned to help set best practices for future Wikipedia-integrated academic publishing. We will be happy to support other publishing groups in introducing their own Wikipediaintegrated article formats.

The versatility of the format also gives wide scope for development of new features. Depending on author demand, we may implement interactive figures, ${ }^{[29]}$ live data, ${ }^{[30]}$ repeated versioned reviews, ${ }^{\left[{ }^{[1]}\right]}$ or the option of double-blind peer review. ${ }^{[32]}$ Indeed, Wiki.J.Med. has already experimented with interactive figures to a limited extent. ${ }^{[33]}$ We also intend to implement greater integration with the structured knowledge database WikiData. As demand increases, one major decision will be whether to split off specialised journals, or stay a large, unified journal with specialised sub-journals. As part of the journal's MediaWiki heritage, these decisions will be made in open discussions with the editor, author, reviewer, and reader communities.

\section{Acknowledgements}

Our thanks to the editorial board of Wiki.J.Med. for their help and advice in setting up their sister journal.

Competing interests: none declared.

\section{References}

1. Shafee, Thomas (2017-11-24). Wikipedia-integrated publishing: a comparison of successful models. 26. Health Inform.. doi:10.13140/rg.2.2.27470.77129.

2. Hodson, Richard. "Wikipedians reach out to academics". Nature. doi:10.1038/nature.2015.18313.

3. Jemielniak, Dariusz; Aibar, Eduard (2016-07-01). "Bridging the gap between wikipedia and academia". Journal of the Association for Information Science and Technology67 (7): 1773-1776. doi:10.1002/asi.23691. ISSN 2330-1643.

4. Shafee, Thomas; Mietchen, Daniel; Su, Andrew I. (2017-08-11). "Academics can help shape Wikipedia". Science 357 (6351): 557-558. doi:10.1126/science.aao0462. ISSN 0036-8075. PMID 28798122.

5. Shafee, Thomas; Masukume, Gwinyai; Kipersztok, Lisa; Das, Diptanshu; Häggström, Mikael; Heilman, James (2017-11-01). "Evolution of Wikipedia's medical content: past, present and future". J Epidemiol Community Health 71 (11): 1122-1129. doi:10.1136/jech-2016-208601. ISSN 0143-005X. PMID 28847845.

6. Allen, Liz; Scott, Jo; Brand, Amy; Hlava, Marjorie; Altman, Micah (2014-0417). "Publishing: Credit where credit is due". Nature 508 (7496): 312-313. doi:10.1038/508312a. 
7. Bould, M. Dylan; Hladkowicz, Emily S.; Pigford, Ashlee-Ann E.; Ufholz, Lee-Anne; Postonogova, Tatyana; Shin, Eunkyung; Boet, Sylvain (201403-06). "References that anyone can edit: review of Wikipedia citations in peer reviewed health science literature". BMJ 348: g1585. doi:10.1136/bmj.g1585. ISSN 1756-1833. PMID 24603564. PMC PMC3944683.

8. Wodak, Shoshana J.; Mietchen, Daniel; Collings, Andrew M.; Russell, Robert B.; Bourne, Philip E. (2012-03-29). "Topic Pages: PLoS Computational Biology Meets Wikipedia". PLOS Computational Biology 8 (3): e1002446. doi:10.1371/journal.pcbi.1002446. ISSN 1553-7358.

9. Su, Andrew I.; Good, Benjamin M.; Wijnen, Andre J. van. "Gene Wiki Reviews: Marrying crowdsourcing with traditional peer review". Gene 531 (2). doi:10.1016/j.gene.2013.08.093.

10. Butler, Declan (2008-12-16). "Publish in Wikipedia or perish". Nature. doi:10.1038/news.2008.1312.

11. Maskalyk, James (2014-10-02). "Modern medicine comes online". Open Medicine 8 (4): e116-e119. ISSN 1911-2092. PMID 25426179. PMC PMC4242788.

12. Jump up $\uparrow$ Izhikevich, Eugene. "Scholarpedia". Scholarpedia 1 (2): 1. doi:10.4249/scholarpedia.1.

13. Giles, Jim (2006). "Wikipedia rival calls in the experts". Nature 443 (7111): 493. doi:10.1038/443493a.

14. Hall, Cody J; Soares da Costa, Tatiana P (2018-06-01). "Lysine: biosynthesis, catabolism and roles". WikiJournal of Science 1 (1): 4. doi:10.15347/wjs/2018.004. ISSN 2470-6345.

15. Chang, Shih Chieh; Bajaj, Saumya; Chandy, K. George (2018-06-01). "ShK toxin: history, structure and therapeutic applications for autoimmune diseases". WikiJournal of Science 1 (1): 3. doi:10.15347/wjs/2018.003. ISSN 2470-6345.

16. Christie, Mike; et al. (2018-06-01). "Radiocarbon dating". WikiJournal of Science 1(1): 6. doi:10.15347/wjs/2018.006. ISSN 2470-6345.

17. Tsirelson, Boris; et al. (2018-06-01). "Spaces in mathematics". WikiJournal of Science 1 (1): 2. doi:10.15347/wjs/2018.002. ISSN 2470-6345.

18. Vandegrift, Guy; Stomel, Joshua (2018-06-01). "A card game for Bell's theorem and its loopholes". WikiJournal of Science 1 (1): 5. doi:10.15347/wjs/2018.005. ISSN 2470-6345

19. Frank, Martin (2013-02-27). "Open but Not Free - Publishing in the 21st Century". New England Journal of Medicine 368 (9): 787-789. doi:10.1056/nejmp1211259.

20. Pulverer, Bernd (2010-11-03). "Transparency showcases strength of peer review". Nature 468 (7320): 29-31. doi:10.1038/468029a.
21. Lawson, Stuart (2015-08-05). "Fee Waivers for Open Access Journals". Publications 3 (3): 155-167. doi:10.3390/publications3030155.

22. Hopewell, Sally; Collins, Gary S.; Boutron, Isabelle; Yu, Ly-Mee; Cook, Jonathan; Shanyinde, Milensu; Wharton, Rose; Shamseer, Larissa et al. (2014-07-01). "Impact of peer review on reports of randomised trials published in open peer review journals: retrospective before and after study". BMJ 349: g4145. doi:10.1136/bmj.g4145. ISSN 1756-1833. PMID 24986891. PMC PMC4077234.

23. Callaway, Ewen; Powell, Kendall (2016-02-16). "Biologists urged to hug a preprint". Nature 530 (7590): 265-265. doi:10.1038/530265a. ISSN 00280836.

24. Callaway, Ewen (2017-02-13). "Heavyweight funders back central site for life-sciences preprints". Nature 542 (7641): 283-284. doi:10.1038/nature.2017.21466. ISSN 0028-0836.

25. Walker, Richard; Rocha da Silva, Pascal. "Emerging trends in peer reviewa survey". Frontiers in Neuroscience 9. doi:10.3389/fnins.2015.00169.

26. Shafee, Thomas; Das, Diptanshu; Masukume, Gwinyai; Häggström, Mikael. "WikiJournal of Medicine, the first Wikipedia-integrated academic journal". WikiJournal of Medicine 4 (1). doi:10.15347/wjm/2017.001.

27. Shen, Cenyu; Björk, Bo-Christer (2015-10-01). "'Predatory' open access: a longitudinal study of article volumes and market characteristics". BMC Medicine 13: 230. doi:10.1186/s12916-015-0469-2. ISSN 1741-7015. PMID 26423063. PMC PMC4589914.

28. "Ethics statement". WikiJournal User Group. Retrieved 2018-03-12.

29. Weissgerber, Tracey L.; Garovic, Vesna D.; Savic, Marko; Winham, Stacey J.; Milic, Natasa M. (2016-08-17). "Correction: From Static to Interactive: Transforming Data Visualization to Improve Transparency". PLOS Biology 14 (8): e1002545. doi:10.1371/journal.pbio.1002545. ISSN 1545-7885. PMID 27533515. PMC PMC4993071.

30. Singh Chawla, Dalmeet (2015-04-22). "'Living figures' make their debut". Nature521 (7550): 112-112. doi:10.1038/nature.2015.17382. ISSN 00280836

31. Mobley, David L.; Zuckerman, Daniel M. (2015-02-03). "A proposal for regularly updated review/survey articles: "Perpetual Reviews"'. arXiv.

32. Okike, Kanu; Hug, Kevin T.; Kocher, Mininder S.; Leopold, Seth S. (201609-27). "Single-blind vs Double-blind Peer Review in the Setting of Author Prestige". JAMA316 (12): 1315. doi:10.1001/jama.2016.11014. ISSN 00987484

33. Shafee, Thomas; Science. "Eukaryotic and prokaryotic gene structure". WikiJournal of Medicine 4 (1): 2. doi:10.15347/wjm/2017.002. 